\title{
Improving the handover and care of acute urological admissions
}

\author{
Edward Bass, Snehal Patel
}

Arrowe Park Hospital, United Kingdom

\begin{abstract}
The point at which a patient is most vulnerable during their journey through the hospital as an acute or emergency admission is the point at which they are transferred to the designated oncoming responsible team. Unsurprisingly, inadequate and incomplete clinical handovers have the potential for latter catastrophic consequences and are utterly avoidable. Recognising these facts, good clinical handover is an essential part of clinical governance and patient safety. Perhaps secondarily, clinical handover - especially when conducted with senior surgical personnel - can be a valuable learning tool for the surgical trainee.
\end{abstract}

An complete audit loop was performed to assess the rate of handover and urology registrar involvement in acute urology periods in 2 month long periods as well as the rate of inadequate investigations and treatment. The interventions introduced included foundation doctor induction training in acute urology cases, an explanation of the importance of handover and a reflective look at ourselves and our approachability. As a result there were significant improvements in the rate of early registrar involvement and successful handover for patients admitted under our take.

\section{Problem}

Whilst working as a core surgical trainee in Urology at my assigned District General Hospital, myself and my supervising specialist registrar became concerned by the standard of practice in the acute urological take. In particular we were concerned that patients triaged to urology by the on call surgical team were not being discussed with a urologist after being reviewed, we were not being informed of their admission and commonly the patients were not handed over to us prior to the day post take.

The aim of this audit is to ascertain whether these concerns are well founded and if so whether or not the situation could be improved.

\section{Background}

The point at which a patient is most vulnerable during their journey through the hospital as an acute or emergency admission is the point at which they are transferred to the designated oncoming responsible team. Unsurprisingly, inadequate and incomplete clinical handovers have the potential for latter catastrophic consequences and are utterly avoidable. Recognising these facts, good clinical handover is an essential part of clinical governance and patient safety. Perhaps secondarily, clinical handover especially when conducted with senior surgical personnel - can be a valuable learning tool for the surgical trainee.

It is recognised that since the introduction of the European Working Time Directive and the subsequent progression to full shift working patterns with minimal overlap between shift times, effective handover of patients admitted during a shift has become increasingly challenging and this is compounded by multi-specialty cover.
Regardless, the aim of clinical handovers is to transfer high quality and appropriate clinical information to the appropriate oncoming healthcare team. A good clinical handover is essential for providing continuation of care, patient safety, and error avoidance. Ultimately, each member of the team should share an understanding of the patients' priorities and convey this to the team accepting responsibility for the patients.

It is recognised that there are common difficulties in delivering effective clinical handover, particularly where the responsibility of multiple surgical specialty admissions out of hours falls on the shoulders of a minimal number of trainees. Invariably, the ultimate responsibility is with the admitting consultant even if it is by name only. However, the increase in shift working makes this singular responsibility impractical. In reality, the whole surgical team is responsible for the continuity of patient care. Effective leadership should demonstrate to each team member their responsibilities as well as direct an understanding of the other team members' roles. An effective clinical handover both reflects and facilitates this.

The aim of this audit was to measure the Trust's compliance with the guidance set out by The Royal College of Surgeons in March 2007 and assess whether or not any inadequacy in our handover practise was leading to poor patient care manifesting in increased morbidity or mortality.

The objectives of the audit were as follows:

1. To assess the prevalence of patients with acute urological diagnoses admitted as inpatients that were inadequately handed over to the relevant responsible team.

2. To assess whether there is a difference between the prevalence of inadequate clinical handovers in and out of hours.

3. To assess whether initial management plans were 


\section{BMJ Quality Improvement Reports}

appropriate in these patients.

4. To assess whether there was an increased risk of morbidity and mortality in these patients - measured by inadequate investigation or treatment.

\section{Baseline measurement}

Methods:

47 randomly selected patients with initial acute urological working diagnoses between 01/06/2014 and 01/07/2014 were included in the retrospective audit. Initially, whether or not the patient had been handed over to the oncoming team at all was recorded. The patients selected were then separated into two groups in regard to whether they were admitted in hours or out of hours. Furthermore, each group was divided by whether or not the Urology registrar was informed and involved in the patient's care prior to handover to the oncoming team. For each patient a review of case notes was performed to assess for a change in working diagnosis after the post-take ward round has taken place and whether the initial investigation or treatment of the patient was inadequate and whether the patient had died. Using the initial admission form and by reviewing the notes, the times taken for an $\mathrm{SHO}$ and then registrar to review the patient were examined. Comparisons between groups' morbidity and mortality could then be made.

Initial Results:

There were 47 acute urological admissions during the audit period. The final diagnosis in $11(23 \%)$ was acute urinary retention with concurrent urosepsis in $4(9 \%)$ patients, in 7 (23\%) renal colic, in 6 (13\%) pyelonephritis, $5(11 \%)$ patients had a simple UTI, and 2 $(5 \%)$ had orchitis. Single patients final diagnoses were urosepsis, a traumatic catheter change, SPC infection, scrotal oedema, renal tumour, pelvic inflammatory disease, an obstructed kidney, a flare of Fowler's syndrome, catheter pain, haematuria, cancer of the prostate, bladder cancer recurrence, and a blocked ureteric stent.

Of the 47 randomly selected patients, $23(49 \%)$ were admitted in hours and $24(51 \%)$ out of hours. Two patients were not examined by an $\mathrm{SHO}$ prior to involvement of the urology registrar. Of those admitted in hours, 13 (59\%) were handed over and the registrar on call was not informed on 10 (44\%) occasions. In hours there was a change of diagnosis in $4(17 \%)$ patients, inadequate investigations in $6(26 \%)$ patients and inadequate treatment in $8(35 \%)$ patients. In those admitted in hours where the registrar on call was informed $13(56 \%)$ patients - $4(17 \%)$ patients had a change in working diagnosis, none were investigated inadequately and a single (4\%) patient received inadequate treatment.

Of the 24 patients admitted out of hours, $9(38 \%)$ were handed over to the oncoming team and the registrar on call was not informed of the admission in $16(67 \%)$ cases. On these occasions there was a change in diagnosis in 8 (33\%) patients, inadequate investigations in $8(33 \%)$ patients, and inadequate treatment commenced in 13 $(54 \%)$ patients. There were $8(33 \%)$ patients admitted out of hours where a Urology registrar or consultant was informed. Of these patients, $2(8 \%)$ had a change of diagnosis, a single (4\%) had inadequate investigations and $2(8 \%)$ had inadequate treatment. No patients died during the course of the audit.

The mean time taken for an $\mathrm{SHO}$ to examine the admitted patient was 3.2 hours (range 0.1 to 11.3 ); the mean time for the registrar to review the patient was 11.4 (range 1.0 to 28.0 ) regardless of when the patient was admitted. In hours, the time prior to being seen by an SHO was 3.1 hours whereas out of hours it was 3.5 hours. The mean average of the time before being seen by a urology registrar in hours was 11.1 hours and out of hours it was 11.3 hours. In cases where the urology registrar was informed of the admission, the mean time before they reviewed the patient was 8.3 hours. In this group the mean time before patients were seen who went on to have inadequate initial investigations was 21.0 hours and for inadequate initial treatment was 12.9 hours. Where registrars were not informed of the admission, the mean time until the patient was seen was 13.5 hours. In this group the mean time before patients were seen who went on to have inadequate initial investigations was 13.4 hours and for inadequate initial treatment was 13.8 hours.

\section{Initial Conclusions}

A similar number of patients are admitted with urological diagnoses both in and out of hours. The majority of these patients have diagnoses of urinary retention, renal colic, and various severities of urinary tract infections.

Occasionally patients are not examined by the on-call SHO prior to being seen by the on-call urology registrar. The range in the time taken for both the $\mathrm{SHO}$ and the registrar to see a particular patient was very large, including one case where the patient had a wait of more than 24 hours to see a urology registrar. To a certain degree this can be explained by the urgency of certain cases, for example where a testicular torsion is suspected or where a supra pubic catheter needs to be passed.

Whether or not a patient receives inadequate care is negatively affected by their time of admission. Patients admitted out of hours are more likely to have a poor initial working diagnosis, are investigated or investigations are planned inadequately and treatment is more likely to be incorrect, inappropriate or missing entirely.

Given that the time a patient waits to see a urology registrar is almost twice as long if they are not informed of the admission as opposed to if they are, we conclude that the huge range in waiting time is greatly influenced by this difference in practise. From our observations this is generally because the patients' details are not changed on the inpatient electronic system and they are 'lost'. Despite this there seems to be no relationship between the time a patient waits to see a urology registrar and whether or not they receive inadequate investigation or treatment.

During the audit period $45 \%$ of patients were handed over to the team taking over the care of the patient following their admission and this was a particular problem out of hours. Likewise, the on call registrar was far less likely to be informed of admissions out of hours when compared to in hours. When a registrar is informed 


\section{BMJ Quality Improvement Reports}

there is less likely to be a change in diagnosis following the post take ward round and a patient is less likely to receive inadequate investigation or treatment before this time. This phenomenon is regardless of the time of admission although morbidity measured in this manner is higher across the board in patients admitted out of hours.

Interestingly, regardless of the time of day a surprising amount of the time the Urology registrar is not informed of patients admitted under their care. This is in stark contrast to the close working relationship between the surgical $\mathrm{SHO}$ and the general surgery registrar. This not only negatively influences patient care by denying them early input from a specialist but also the junior surgeon's educational development.

From this data we can gather that the handover from the general surgical take to the accepting urological team is neither adequate nor occurring with reasonable frequency. Patients are less likely to receive specialist input to their acute care when out of hours and these patients are less likely to be handed over. This often results in further delays before they are seen by a urologist and correct interventions are enacted.

See supplementary file: ds5093.jpg - "cycle 1"

\section{Design}

Intervention

Much had already been improved dramatically by the introduction of a dedicated on call urology registrar rota between 08:00 and 23:00. This has allowed far easier access to specialist input and advice in these hours. Of course, we viewed this team to team relationship as reciprocal and as such the urology registrars were encouraged to have a more obvious presence on the Surgical Assessment Unit, particularly between 5 and $9 \mathrm{pm}$.

In addition we organised sessions with the oncoming junior surgical staff to train them in acute urological emergencies and highlight the importance of handover in relation to patient safety and essentially we now demand that this happens on two occasions daily.

Monitoring the benefits of these changes was organised for 12 months later with a repeat of the audit using the same parameters. The audit committee received a report with our recommendations and action plan.

\section{Strategy}

\section{PDSA Cycle 1}

In order to improve the low rate of early involvement in our patients and the number of them which ultimately were not handed over or suffered increased morbidity, my colleague organised a session with the incoming group of junior doctors to explain and emphasise the importance of seeking our specialist involvement early. The session was well received and the new junior doctors left with a greater understanding that we are an approachable speciality and that early specialist involvement is good for the patients and for their training.

\section{PDSA Cycle 2}

There was a perception that the junior surgical on call doctors had little previous exposure to, or training in urology and as such were underprepared to properly manage the acute cases which presented themselves. To address this, a teaching session covering the common urological emergency case mix was organised as part of the junior doctors induction. It was fed back to us that this training was appreciated as the regional medical school which inevitably supplies the vast majority of Foundation trainees to the hospital provides little exposure to Urology which they felt was surprising given the size of the specialty.

PDSA Cycle 3

Additionally, the results of this audit cycle were fed back to the middle grade urology staff with a view to improving our own presence on the acute surgical wards. In particular we aimed to have a strong presence between 5 and $9 \mathrm{pm}$ on the surgical assessment unit and during this time we should be reviewing the day's take. This would ensure two post take ward rounds every 24 hours. Ultimately the middle grade doctors agreed that this should be the standard of care and the approach was adopted with relative ease.

\section{Results}

The post intervention measurement was performed in the same manner as the pre intervention measurement.

Methods

45 randomly selected patients with initial acute urological working diagnoses between 01/02/2015 and 01/03/2015 were included in the retrospective audit. Initially, whether or not the patient had been handed over to the oncoming team at all was recorded. The patients selected were then separated into two groups in regard to whether they were admitted in hours or out of hours. Furthermore, each group was divided by whether or not the Urology registrar was informed and involved in the patient's care prior to handover to the oncoming team. For each patient a review of case notes was performed to assess for a change in working diagnosis after the post-take ward round has taken place and whether the initial investigation or treatment of the patient was inadequate and whether the patient had died. Using the initial admission form and by reviewing the notes, the times taken for an $\mathrm{SHO}$ and then registrar to review the patient were examined. Comparisons between groups' morbidity and mortality could then be made.

Results

There were 45 acute urological admissions during the audit period. The final diagnosis in $6(14 \%)$ was acute urinary retention with concurrent urosepsis in $8(19 \%)$ patients, in $2(5 \%)$ renal colic, in 3 


\section{BMJ Quality Improvement Reports}

(7\%) pyelonephritis, 4 (10\%) patients had a simple UTI or hydronephrosis, $2(5 \%)$ had urosepsis, $2(5 \%)$ had a blocked nephrostomy, and $2(5 \%)$ had an infected ureteric stent. Single patients final diagnoses were cellulitis, back pain, haematuria, obstructed-infected kidney, prostatitis, PUJ obstruction, and a renal abscess.

Of the 45 randomly selected patients, $22(49 \%)$ were admitted in hours and $23(51 \%)$ out of hours. Of those admitted in hours, 18 $(82 \%)$ were ultimately handed over but the registrar on call was not informed on 8 (36\%) occasions. In this group, in hours there was a change of diagnosis in $5(23 \%)$ patients, inadequate investigations in $4(18 \%)$ patients and inadequate treatment in $4(18 \%)$ patients. In those admitted in hours where the registrar on call was informed $14(64 \%)$ patients - $7(32 \%)$ patients had a change in working diagnosis, none were investigated inadequately and none received inadequate treatment.

Of the 24 patients admitted out of hours, $22(96 \%)$ were handed over to the oncoming team however, the registrar on call was not informed of the admission in $6(26 \%)$ cases. On these occasions there was a change in diagnosis in $3(13 \%)$ patients, inadequate investigations in $1(4 \%)$ patient, and inadequate treatment commenced in $2(9 \%)$ patients. In those admitted out of hours where the registrar or consultant on call was informed - 17 (74\%) there was a change in diagnosis in $4(36 \%)$ patients, inadequate investigations in a single patient (4\%), and inadequate treatment in $3(13 \%)$. No patient died following admission during the course of this audit.

The mean time taken for an $\mathrm{SHO}$ to examine the admitted patient was 4.4 hours (range 0.7 to 24.0); the mean time for the registrar to review the patient was 11.4 (range 1.0 to 28.0) regardless of when the patient was admitted. In hours, the time prior to being seen by an $\mathrm{SHO}$ was 4.6 hours whereas out of hours 4.3 hours. The mean average of the time before being seen by a urology registrar in hours was 10.6 hours and out of hours it was 11.7 hours. In cases where the urology registrar was informed of the admission, the mean time before they reviewed the patient was 9.3 hours. In this group the mean time before patients were seen who went on to have inadequate initial investigations was 11.7 hours and for inadequate initial treatment was 8.6 hours. Where they were not informed of the admission, the mean time until the patient was seen was 15.6 hours. In this group the mean time before patients were seen who went on to have inadequate initial investigations was 19.2 hours and for inadequate initial treatment was 13.8 hours.

\section{Data Analysis}

Major improvements were made following the introduction of the interventions. The percentage of patients admitted in hours ultimately handed over to the take team improved from $60 \%$ to $82 \%$. Additionally, the percentage of patients admitted out of hours ultimately handed over to the take team improved from $38 \%$ to $95 \%$. The percentage of patients admitted in hours where the on call urology registrar was informed of their admission improved from $57 \%$ to $64 \%$. Finally, the percentage of patients admitted out of hours where the on call urology registrar was informed of their admission improved from $33 \%$ to $74 \%$. These improvements were all statistically significant (Fisher's exact tests $p<0.05$ ).

The time that elapses prior to being seen by both the on call $\mathrm{SHO}$ and urology registrar remains largely the same. Likewise, in the same manner as audit cycle 1, the longer it takes for a patient to be seen by a registrar, the more likely that they suffer morbidity particularly if the on call registrar is not informed of their admission.

See supplementary file: ds5094.jpg - "Cycle 2"

\section{Lessons and limitations}

We learnt a number of lessons from carrying out this project. Most importantly was that although initially we developed negative hypotheses regarding why we were not being involved in the care of our patients early in their inpatient journey, it turned out that junior staff did not see us as approachable and as such did not contact us often. Without doubt, this is a shame and thankfully was easily remedied. Indeed, this problem exacerbated the next. That because the junior staff had little experience in urology prior to starting their careers and then tended not to work with us in the management of patients they were not developing their knowledge and skills for the future.

Without doubt these problems were easily rectified, however, they will require at least yearly sessions with new junior doctors as part of induction and in all likelihood, more often than that.

Naturally the project had it's limitations. Although prospective the sample sizes were small and the audit period for both cycles was short. As such the slightly startling improvements may be overly reaching however, the general feeling is that that there has been improvement in our service provision. Regardless, in the near future the department will be introducing an 'Consultant of the Week' programme and following that a third longer cycle will be performed to further assess for improvements.

\section{Conclusion}

The 3 simple changes made to our practice both inwardly and outwardly have made great improvements to the care of our patients. Our handovers are more successful and the middle grade urologists are involved in the care of their patients at a much earlier stage much more commonly. The result of this is our patients are at a reduced risk of harm than they were 12 months ago although in absolute terms this has been difficult to quantify given the limits of the study.

\section{References}

1. Safe handover: guidance from the working time directive working party. http://www.rcseng.ac.uk/publications/docs: RCSE Association BM. 2007

2. Safe handover, safe patients: guidance on clinical handover for clinicians and managers: British Medical Association. 2004 
BMJ Quality Improvement Reports

\section{Declaration of interests}

The authors have no conflicts of interest to declare

\section{Acknowledgements}

Mr Snehal Patel - Supervising Consultant Urologist at Wirral University Hospital Trust

\section{Ethical approval}

This study was deemed a clinical improvement project and therefore did not require local ethical approval. 